\title{
APPLICATION OF A LATTICE GAS MODEL FOR SUBPIXEL PROCESSING OF LOW-RESOLUTION IMAGES OF BINARY STRUCTURES
}

\author{
ZBISŁAW TABOR \\ Department of Image Analysis, Institute of Applied Computer Science, Cracow University of Technology, Al. \\ Jana Pawła II 37, 31-864 Cracow, Poland \\ e-mail: tabor@alphas.if.uj.edu.pl \\ (Accepted October 17, 2006)
}

\begin{abstract}
In the study an algorithm based on a lattice gas model is proposed as a tool for enhancing quality of lowresolution images of binary structures. Analyzed low-resolution gray-level images are replaced with binary images, in which pixel size is decreased. The intensity in the pixels of these new images is determined by corresponding gray-level intensities in the original low-resolution images. Then the white phase pixels in the binary images are assumed to be particles interacting with one another, interacting with properly defined external field and allowed to diffuse. The evolution is driven towards a state with maximal energy by Metropolis algorithm. This state is used to estimate the imaged object. The performance of the proposed algorithm and local and global thresholding methods are compared.
\end{abstract}

Keywords: image processing, Ising model, trabecular bone.

\section{INTRODUCTION}

For the most common types of image acquisition devices, pixels of an acquired image represent an averaging of the signal across a finite area of the scene or specimen. If the imaged object is a binary structure (i.e., structure built of two distinct phases), image pixels, which straddle a boundary between two phases average their brightness and have an intermediate intensity, depending on the fraction of both phases contained in a pixel.

Frequently the resolution of an acquired image is not sufficient to extract all interesting features of the object. In such a case super-resolution algorithms (Park et al., 2003) can be applied to enhance the resolution. It is crucial, that the super-resolution algorithms require at input multiple low-resolution images, which differ from one another because of the object movement or exposition conditions. The number of scenes on which any super-resolution algorithm operates depends on the size of the smallest feature, which has to be extracted.

The central problem of any algorithm enhancing resolution of the images of binary structures is, how the interface between two phases should be drawn across the relatively large in size pixels of the acquired image. In this paper it is demonstrated that a lattice gas model can be a useful tool to address the above formulated problem. The proposed method requires one low-resolution input image to produce its enhanced (in a sense of decreased pixel size) representation. Strictly speaking, super-resolution can not be achieved with the developed algorithm under arbitrary conditions, because the information contained in the enhanced image is the same as in the acquired one. However, if the phase interface can be described by a suitably low-dimensional family of functions, improvement of the quality of the low-resolution data can be achieved by suitable subpixel processing, which however is not superresolution restoration in a strict sense (no subpixel features exist if all information is already present in the low-resolution image).

\section{PROBLEM FORMULATION}

It is assumed that low-resolution image is an $\mathrm{M} \times \mathrm{M}$ matrix of integer numbers, taking values (gray-level intensities) in the range from 0 to L. 0 and $\mathrm{L}$ are intensities of the "black" and "white" phases, respectively. The restored image (the estimate of the object, which has to be reconstructed), is an $\mathrm{M} \cdot \mathrm{N} \times \mathrm{M} \cdot \mathrm{N}$ matrix $(\mathrm{N}>1)$, elements of which are either 0 (black phase) or 1 (white phase). Because image acquisition could be represented as an averaging of the signal across a finite area of the scene, the restored image is one of the possible solutions to the following set of $\mathrm{M}^{2}$ equations: 


$$
\sum_{i, j=0}^{N-1} p_{I+i, J+j}=(\text { int }) \frac{N^{2} \cdot G_{l, J}}{L},
$$

where $G_{I, J}$ is the gray level intensity of the (I,J)-th pixel of the low-resolution image and $\mathrm{M}^{2} \cdot \mathrm{N}^{2}$ unknown $\mathrm{p}_{\mathrm{I}+\mathrm{i}, \mathrm{J}+\mathrm{j}}$ are the 0 or 1 values attributed to the $(\mathrm{I}+\mathrm{i}, \mathrm{J}+\mathrm{j})-$ th pixel of the restored image.

All information contained in the acquired image is used to write Eq. 1. To enable the solution, prior assumptions are necessary. In particular, if it is assumed that phase interface of the object can be parameterised by suitably low-dimensional family of functions (the phase interface is sufficiently smooth), then super-resolution could be achieved. More formally, the number $\mathrm{K}$ of parameters, describing phase interface must be less than $\mathrm{M}^{2}$. If $\mathrm{K}>>\mathrm{M}^{2}$ superresolution is not possible.

Indeed, assume that a phase interface within a pixel of a low-resolution image can be represented by a straight line. Then, for any selected slope of the straight line segment, intercept can be calculated from Eq. 1, which determines the fraction of white and black phases within a pixel of a low-resolution image. The slope can be determined from the demand that the phase interface at the border of neighbouring pixels is continuous. In such a case Eq. 1, together with the assumption about simplicity of the phase interface deliver sufficient information to reproduce an object. Having specified some data set, the validity of the above assumption must be tested by suitable comparison of known objects and their restored images. Here I present an algorithm for subpixel processing of images of binary structures. The assumption about smoothness of the phase interface is not necessary for the algorithm to work. Conversely, satisfactory performance of the algorithm suggests that this assumption is fulfilled at least approximately.

As an example of a binary structure, trabecular bone is considered in the present study. Trabecular bone is especially interesting porous material, because it develops under the control of a living organism (Tabor et al., 2002), it is highly optimized, comparing its strength to its density and adapts to varied load conditions (Huiskes et al., 2000). Osteoporosis, which is a common trabecular bone pathology is a serious health and economic problem. High resolution images (pixel size significantly smaller than typical thickness of trabeculae, which is of the order of $100 \mu \mathrm{m}$ ) are necessary for reliable quantification of the trabecular structure (van Rietbergen et al., 1995; Müller et al., 1996; Ulrich et al., 1998), while only low-resolution images of trabecular bone are clinically available (pixel size of the order of $150 \mu \mathrm{m}$ ), because on the limitation on applied dose of X-rays and acquisition time.

High-resolution (HR) images of vertebral trabecular bone were obtained from 16 autopsy cases, from which the third lumbar vertebral body was removed. The procedure of bone specimens preparation and image acquisition was described elsewhere (Tabor and Rokita, 2000) and only brief details are presented here. The vertebral bodies were sectioned perpendicularly to the vertical axis of the vertebra and ground to a uniform thickness of $200 \mu \mathrm{m}$. 8-bit images of each section were acquired using a lowmagnification digital camera. The pixel size of the images was equal to $30 \mu \mathrm{m}$. In each case the brightness histograms contained two well separated peaks and the bone/marrow interfaces were selected by setting the threshold level between the peaks. Sample binary image of a histological sections of vertebral trabecular bone is shown in Fig. 1. Because resolution of the order of $30 \mu \mathrm{m}$ is sufficient for the typical histomorphometric measurements (Müller et al., 1996), the images are treated as the objects, i.e. it is assumed that they contain all interesting information. Low-resolution (LR) images of the objects were simulated by averaging pixels of the objects over $10 \times 10$ pixel boxes. A result of the averaging procedure $(51 \times 51$ pixel grey level images, $300 \mu \mathrm{m}$ pixel size) performed for the section depicted in Fig. 1 is shown in Fig. 2.

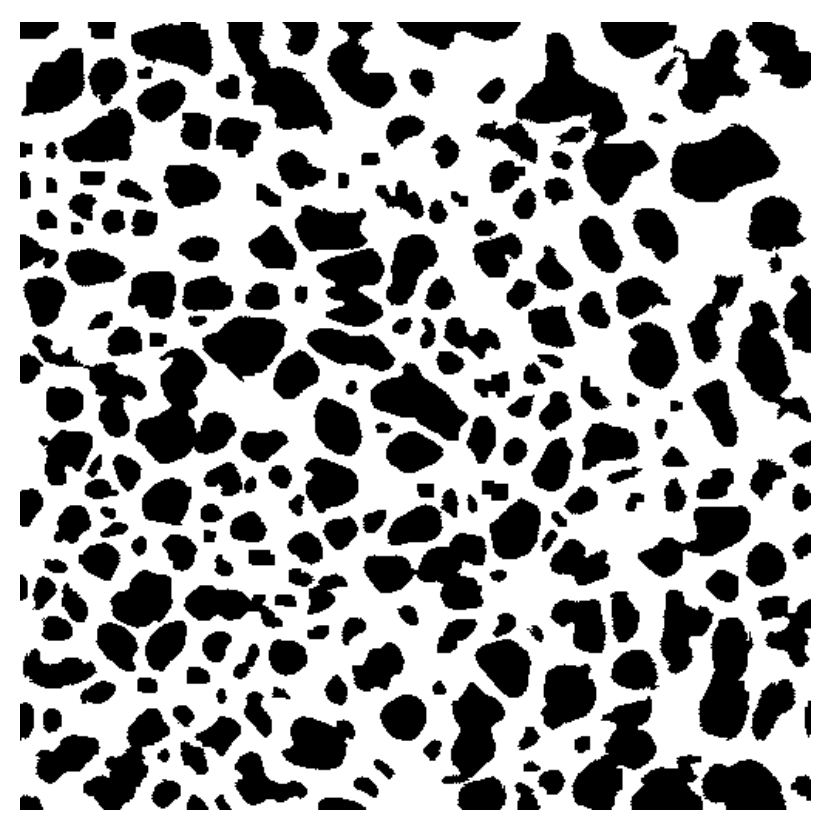

Fig. 1. A sample high-resolution binary image of a sections of vertebral trabecular bone (pixel size $30 \mu \mathrm{m}$ ). 


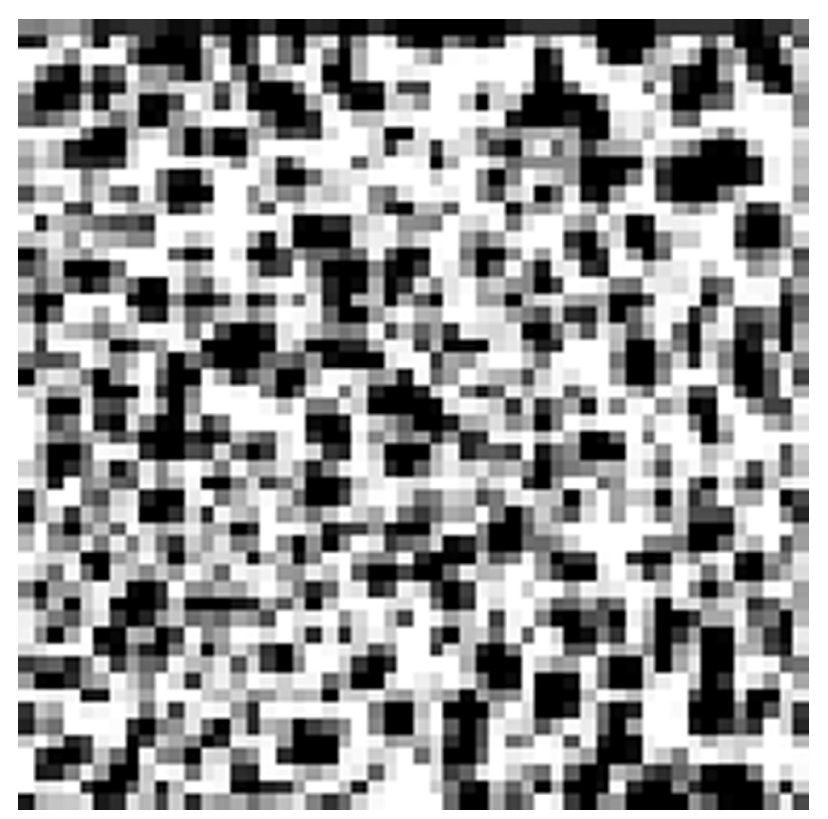

Fig. 2. A low-resolution image of the structure shown in Fig. 1 (300 um pixel size). The range of grey levels present in the image is due to the partial volume effect.

\section{IMAGE RECONSTRUCTION}

\section{Pixel subdivision and ridge images}

The final goal of a resolution enhancement algorithm is to draw the phase interface across the interior of the pixels of the LR images. At the first stage of the algorithm, the first guesses of the restored images are created. For this purpose each pixel of a LR image is replaced by an $\mathrm{N} \times \mathrm{N}$ matrix, $\mathrm{N}^{2} \cdot \mathrm{G} / \mathrm{L}$ elements of which are set to 1 and the rest to 0 (where $\mathrm{G}$ is the grey level of the replaced pixel). In Fig. 3 a $10 \times 10$ matrix replacement of a part of the structure shown in Fig. 2 is shown.

To find the ridges of the white phase, a bicubic interpolation $\mathrm{M} \cdot \mathrm{NxM} \cdot \mathrm{N}$ image is obtained from each corresponding LR image. Then the ridge detection procedure (Ross, 1994; Elmoutaouakkil et al., 2002) is applied to the interpolated images. In Fig. 4 a bicubic interpolation image of the image shown in Fig. 1 is depicted, together with the white phase ridges.

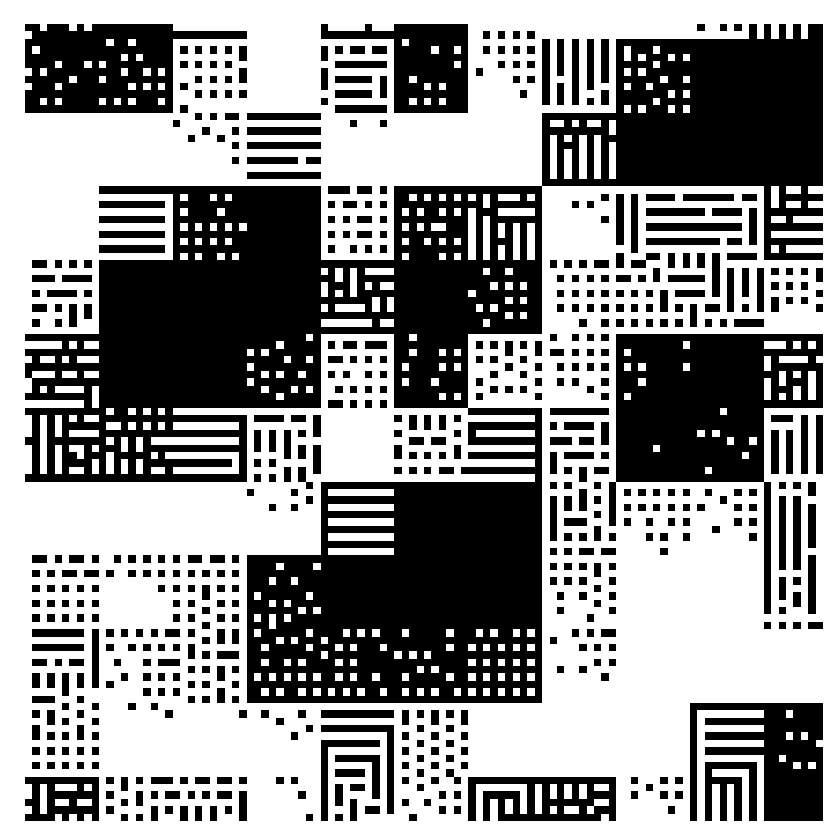

Fig. 3. A first guess particle model binary image - the result of transformation of a part of the image depicted in Fig. 2 (30 $\mu$ m pixel size).

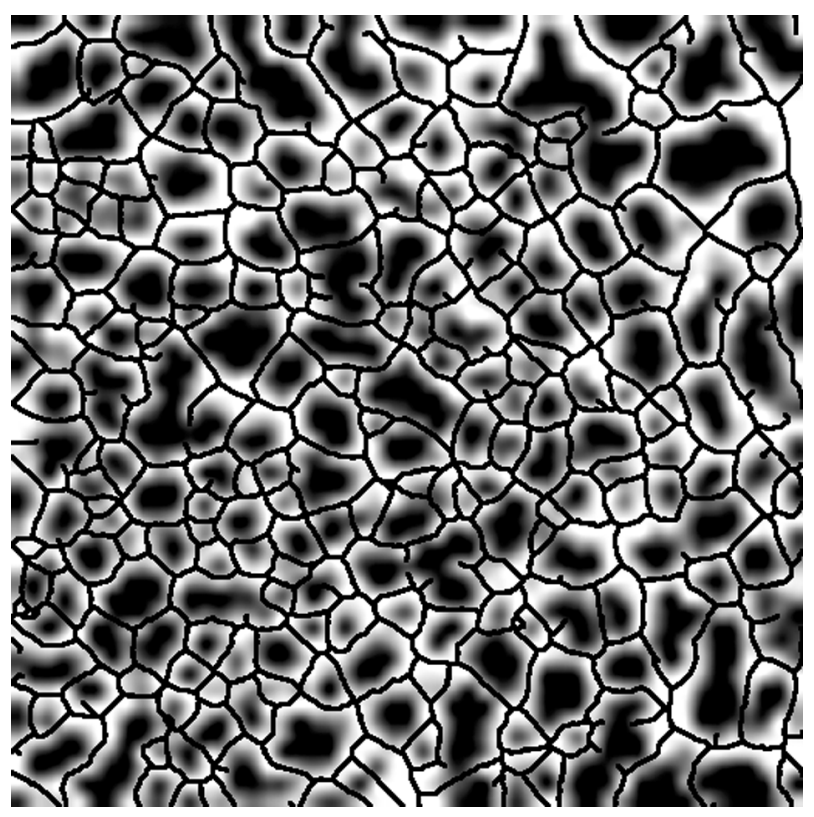

Fig. 4. An image (30 um pixel size) obtained by bicubic interpolation of the image shown in Fig. 2. The image is used to determine the ridges of the white phase. The ridges are drawn as black lines. 


\section{Lattice gas model}

The following method is introduced to assign black and white labels to the pixels of enhanced image. The assumption about smoothness of the phase interface leads to a model, according to which each white pixel of the first guess image is identified with a particle allowed to diffuse inside the $\mathrm{N} \times \mathrm{N}$ matrix, to which the pixel belongs. It is further assumed that the particles interact with one another. The interaction is chosen to be Ising-like. Besides self-interaction, particles interact also with an external field. This interaction is defined as attraction to the ridges of the white phase. Thus the energy of the system is defined as follows:

$$
E=\alpha_{1} \sum_{<i, j>} w_{i} \cdot w_{j}-\alpha_{2} \sum_{i} d\left(w_{i}\right),
$$

where $\alpha_{1}$ and $\alpha_{2}$ are some parameters, $\mathrm{w}_{\mathrm{i}}$ is set to 1 for white pixels and 0 for black pixels. The second term of the energy (2) is responsible for aligning the phase interface along the white phase ridges, i.e., it specifies locally the slope of the phase interface. The first term ensures that the phase interface is smooth.

I have found it experimentally, that for $\mathrm{N}=10$ two-pixel range of the self-interaction is an optimal choice. $\mathrm{d}\left(\mathrm{w}_{\mathrm{i}}\right)$ denotes the distance of $\mathrm{w}_{\mathrm{i}}$ from the nearest ridge. The distance field was computed only once, at the beginning of the simulation, using the digital distance transform (Ross, 1994).

The diffusion is driven by standard Metropolis algorithm towards a state with maximal energy. I choose $\alpha_{2}$ a few times larger than $\alpha_{1}$ (e.g. $\alpha_{1}=2, \alpha_{2}=$ $15)$ and thus there is much higher penalty for increasing the distance from the ridge, than for decreasing the number of neighbors. Thus I can split the maximization procedure in two parts. First, I maximize the distance part of the energy with the self-interaction term turned-off $\left(\alpha_{1}=0\right)$. Typically a few dozens of iterations (a single iteration consisting of attempts to change the state of each particle) are necessary to complete this step. Then to make phase interface smooth I maximize the self-interaction term, with the distance term turned-off, but now I allow the particles to move in more broad boxes (not larger than $20 \times 20$ pixels for $\mathrm{N}=10$ ). This step also requires a few dozen of iterations. Every iteration takes about one second for an image $500 \times 500$ pixels on a PC equipped with a Pentium IV, $2.4 \mathrm{GHz}$ processor. Thus the maximization procedure takes not more than two minutes for a single image $500 \times 500$ pixels. The values of the distance term energy, obtained during reconstruction of the structure shown in Fig. 1 are plotted vs. the iteration number in Fig. 5a. The values of the self-interaction term energy, obtained for the same structure are plotted vs. the iteration number in Fig. 5b.

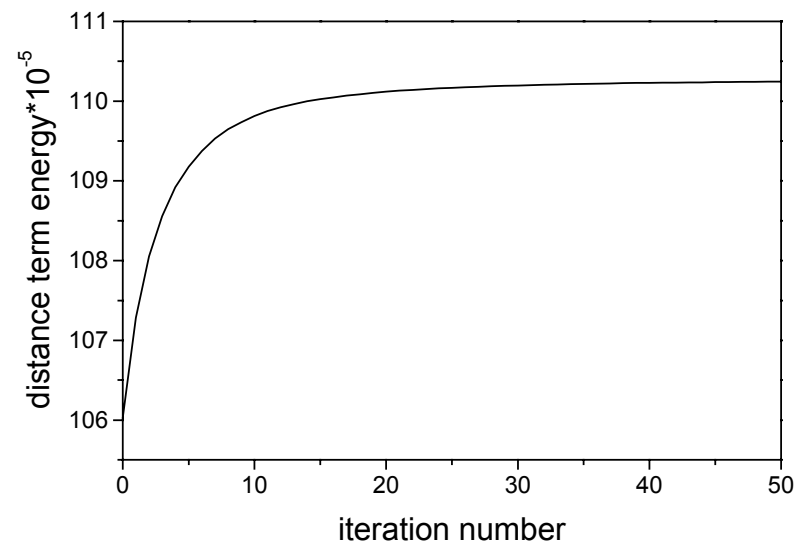

(a)

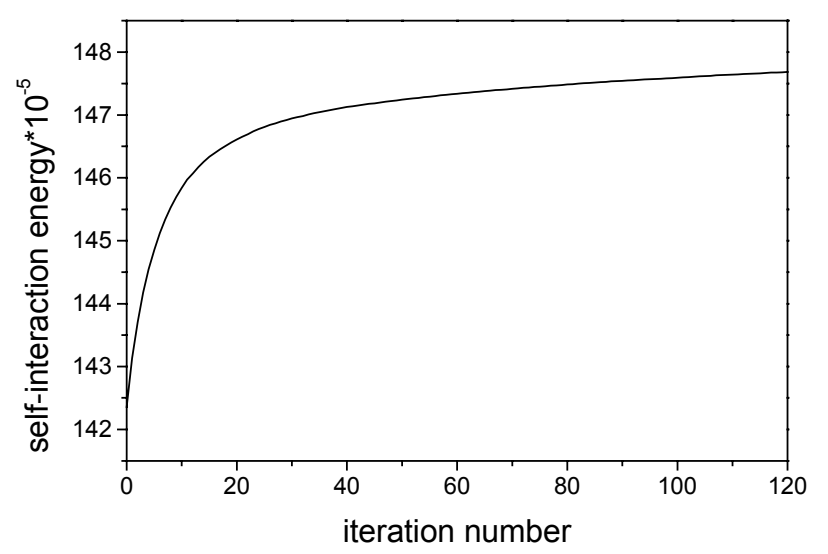

(b)

Fig. 5. The evidence of the convergence of the distance term energy (a) and self-interaction term energy (b), during the maximization procedure.

\section{Pruning}

In the final step of the processing the images are cleaned to remove clusters of black or white pixels (Fig. 6) smaller than some cut-off value (typically 25 pixels). Such isolated clusters of white or black pixels can arise due to noise added to the image or due to the diffusion to the border of boxes during the maximization of the distance term energy. The result of the reconstruction of the object depicted in Fig. 1, based on the information contained solely in Fig 2 is shown in Fig. 7. 


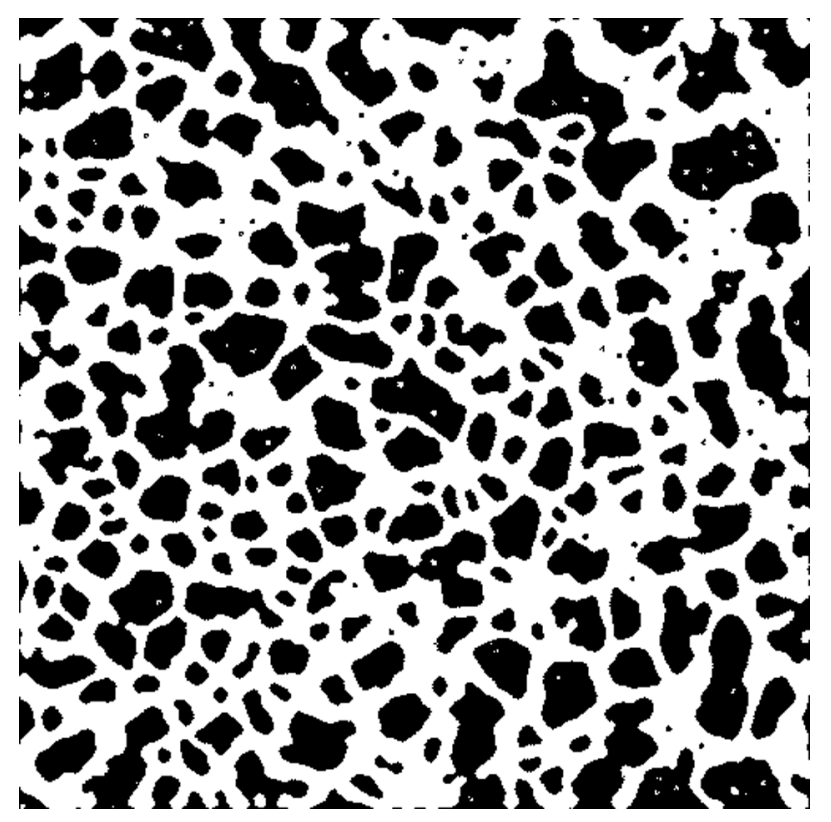

Fig. 6. A binary image (30 $\mu$ m pixel size), which is the results of maximization of the energy defined in Eq. 2 can contain isolated clusters of white or black pixels (especially in the presence of noise).

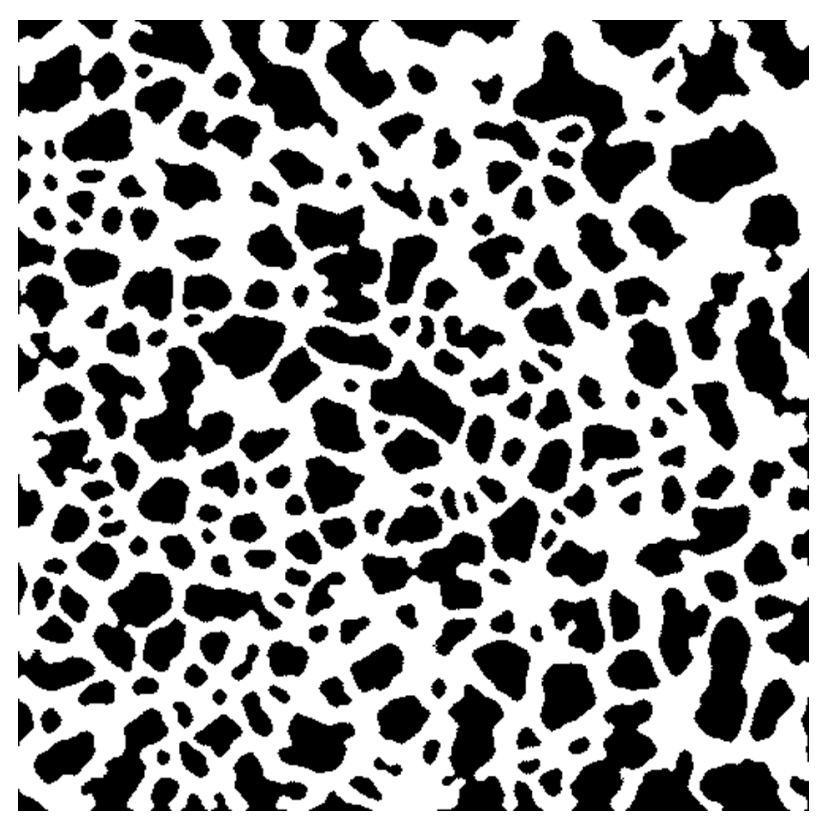

Fig. 7. A binary images after pruning operation (30 um pixel size) is the final reconstruction of the original image, depicted in Fig. 1. The reconstruction, based on the novel algorithm, uses only the data present in Fig. 2.

\section{PERFORMANCE}

The performance of the introduced algorithm was compared with standard thresholding techniques applied to:

1. Globally thresholded bicubic interpolation images: in this case the threshold value is set to achieve the fraction $\mathrm{BV} / \mathrm{TV}$ of white pixels of the thresholded image equal to the BV/TV of the original image (Fig. 8).

2. Locally thresholded bicubic interpolation images: in this case the threshold is set locally in $\mathrm{n} \times \mathrm{n}$ matrices of pixels to achieve local BV/TV (i.e., $\mathrm{BV} / \mathrm{TV}$ evaluated for nxn matrix of pixels) of the thresholded image equal to the corresponding local $\mathrm{BV} / \mathrm{TV}$ of the original image. $\mathrm{n}$ in the range from 10 to 50 was used in the calculations $(n=50$ shown in Fig. 9).

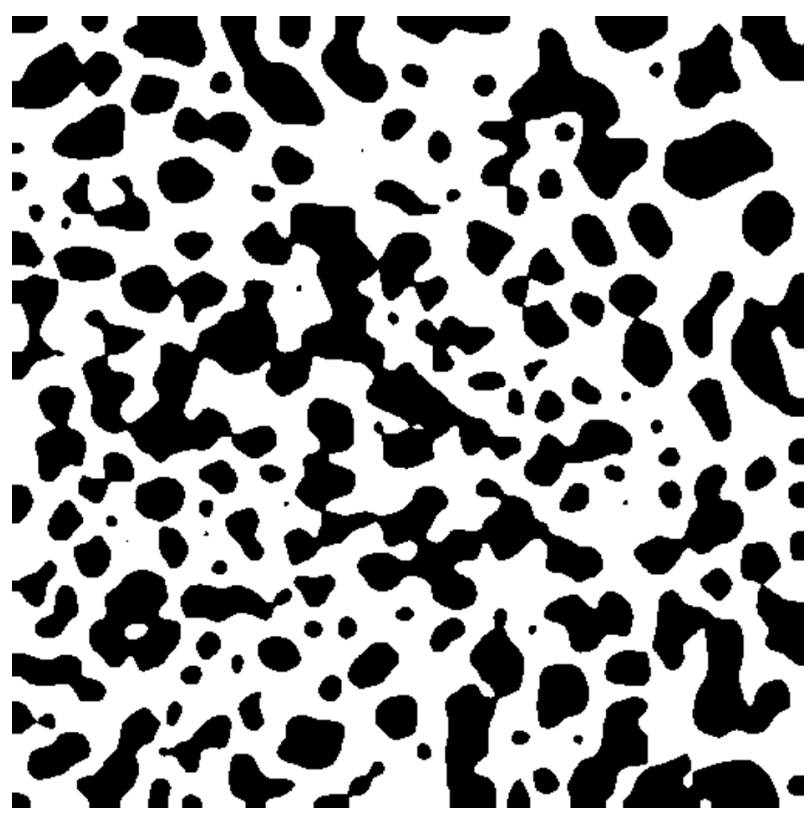

Fig. 8. A binary image (30 $\mathrm{mm}$ pixel size), which is the result of global thresholding of the image depicted in Fig. 4. The threshold value is set to achieve $B V / T V$ of the thresholded image equal to the $B V / T V$ of the image depicted in Fig. 1. 


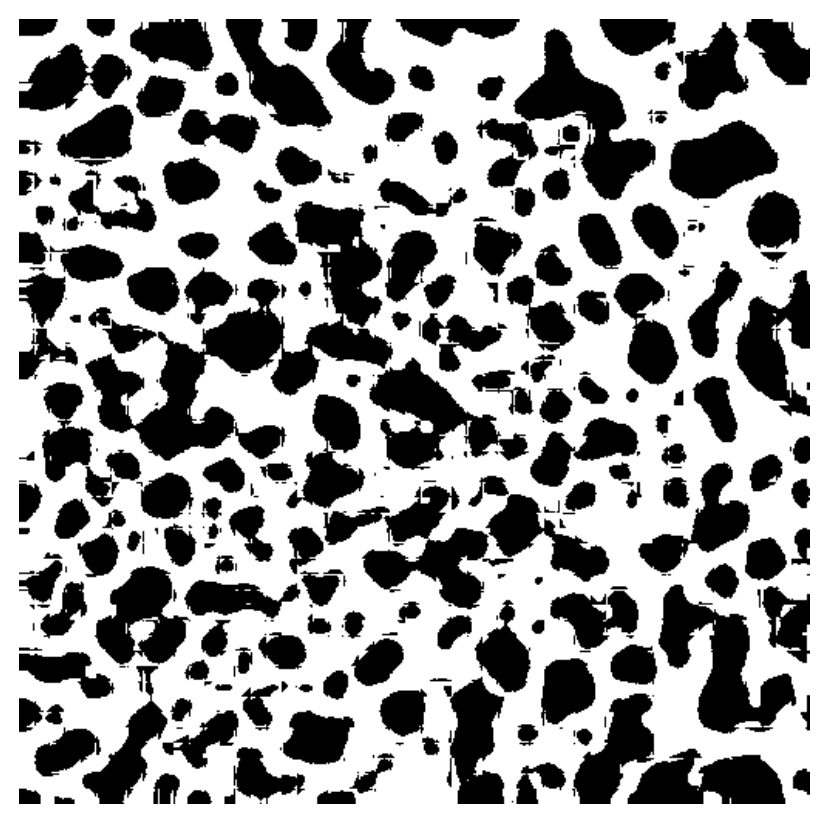

Fig. 9. A binary image (30 $\mu$ m pixel size), which is the result of local thresholding of the image depicted in Fig. 4. The threshold value is set locally in $50 \times 50$ matrix of pixels to achieve local BV/TV of the thresholded image equal to the corresponding local $B V / T V$ of the image depicted in Fig. 1.

Following quantities were used to test the performance of the introduced algorithm vs. the performance of standard thresholding procedures:

1. Mean pore area P.Ar.

2. Euler number density E.N.

3. Mean thickness of white phase Wh.Th.

4. Mean thickness of black phase B.Th.
5. The badly reconstructed area - the half of the number of white pixels of the image being the difference between the original image and the reconstructed image, related to the number of white pixels of the original image. The pixel of a difference image is white if pixels of subtracted binary images are different and it is black otherwise.

6. Mean area of bad clusters: a badly reconstructed pixel is any pixels of the reconstructed image, which is white, while the corresponding pixel of the original image is black. Badly reconstructed pixels are grouped in clusters. The reconstruction errors are smaller if the mean area of bad clusters is lower.

The results of comparison (mean value, standard error) are summarized in Table 1. The introduced method allows quantifying the reconstructed structure with errors smaller than thresholding techniques. This is true for both structural parameters (P.Ar, E.N, Wh.Th and B.Th) and measures of badly reconstructed area. The advantages of the presented algorithm are especially noticeable in the cases, when porous structure with low thickness of the white phase has to be restored from a low-resolution image. An example is presented in Fig. 10. The badly reconstructed area is equal to $5 \%, 21 \%$ and $19 \%$ for lattice gas model, global and local thresholding, respectively. The main drawback of the thresholding method is that they do not recover the connectivity of the original structure. The Euler number of the original structure is equal to -126. The Euler number for lattice gas model, global and local thresholding is equal to $-108,26$ and 90 , respectively.

Table 1. The comparison of the performance of the novel algorithm vs. threshold techniques.

\begin{tabular}{lllll}
\hline Parameter & Original images & Simulation & Global threshold & Local threshold \\
\hline P.Ar $\left[\mathrm{mm}^{2}\right]$ & $1.04(0.14)$ & $1.11(0.16)$ & $2.07(0.33)$ & $0.66(0.10)$ \\
E.N $\left(1 / \mathrm{mm}^{2}\right]$ & $-68(6)$ & $-67(7)$ & $-33(5)$ & $-72(11)$ \\
Wh.Th $(\mu \mathrm{m})$ & $280(15)$ & $279(15)$ & $354(17)$ & $242(14)$ \\
B.Th $(\mu \mathrm{m})$ & $319(19)$ & $321(20)$ & $407(25)$ & $282(19)$ \\
badly reconstructed area [\%] & - & $3.1(0.2) \%$ & $9.9(0.9) \%$ & $8.0(0.9) \%$ \\
mean area of bad clusters $[\mathrm{pixel}]$ & - & $3.8(0.1)$ & $10.3(0.7)$ & $5.4(0.4)$
\end{tabular}



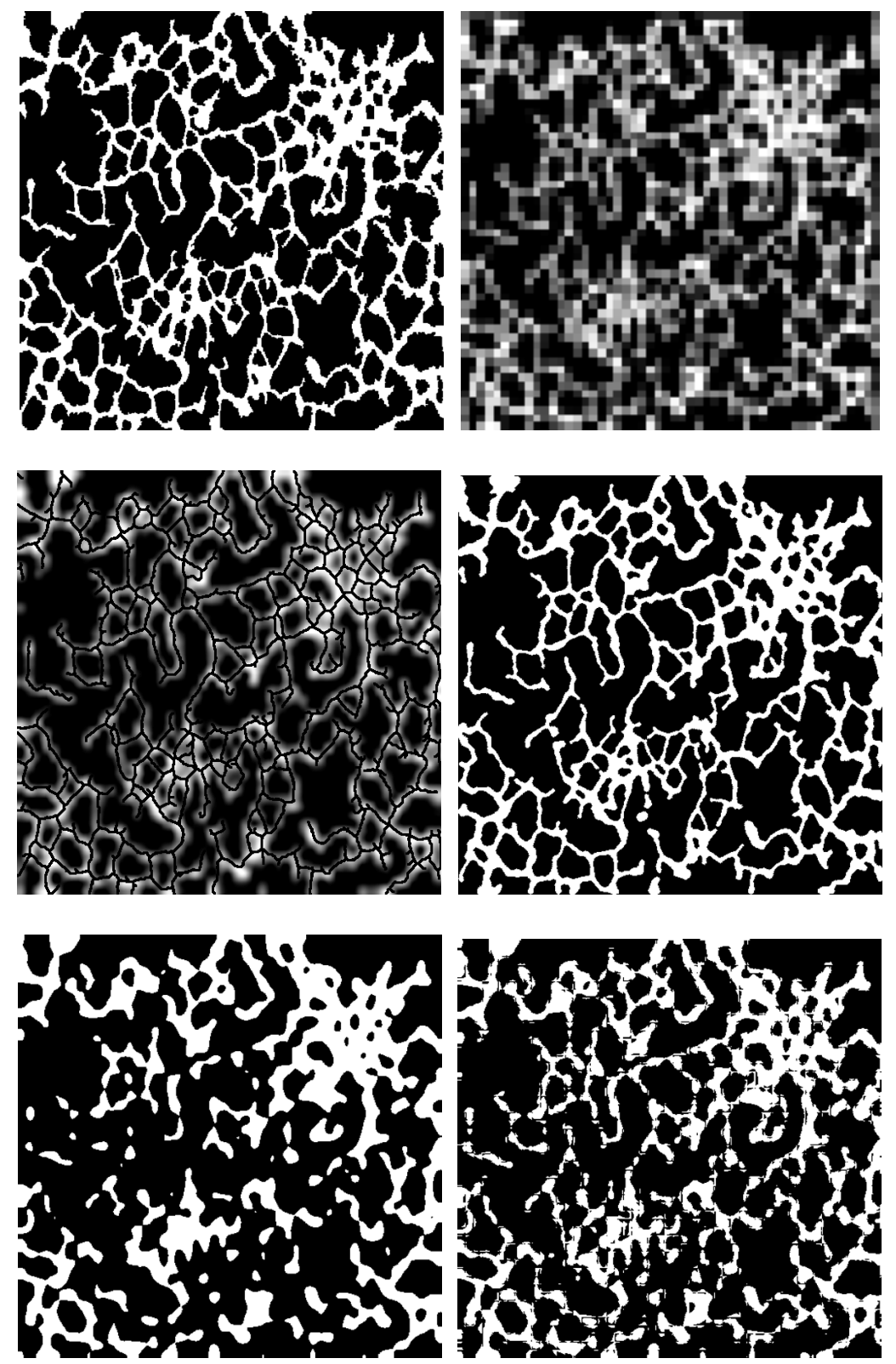

Fig. 10. A high- (top-left) and low-resolution (top-right) image of a low-thickness structure, its ridges (middleleft), lattice gas model (middle-right), global (bottom-left) and local (bottom-right) thresholding restoration.

\section{DISCUSSION}

There are at least two reasons, why thresholding methods lead to relatively poor results. The first reason is significant variation of the thickness of the white phase (trabeculae) over the analyzed field of view. Generally, thick trabeculae are properly recognized as white phase. With decreasing trabcular thickness it becomes more problematic to properly recognize trabeculae. This is clearly seen from the results of the measurement of the Euler number. Small value of E.N for global threshold is just the result of the described process. In the case of the local threshold, E.N is close to E.N of the original sections but it is due to the existence of small loops - artifacts of the local threshold method - which compensate the lack of trabeculae. Thresholding methods lose trabeculae, because they do not "feel" the presence of trabecular ridges, what is the second reason, why these methods produce large restoration errors. Exactly for this reason the distance term in the energy (Eq. 2) is set much more important, by appropriate choice of the parameters $\alpha_{1}$ and $\alpha_{2}$. Diffusion with particle self-interaction term only does not lead to object reconstruction of acceptable quality. 
The results presented above were obtained for idealized case of no noise imposed on the lowresolution data. Extensive calculation were also performed for the case of Gaussian noise added to the low-resolution data (signal-to-noise ratio from 30 to $40 \mathrm{~dB}$ ). In this case reasonable restoration of an object was possible if the input low-resolution images of the objects were an average over multiple (typically not more than two dozens) low-resolution noisy expositions.

From the comparison of the object and restored images (e.g. Fig. 10, Fig. 1 and Fig. 7) it is evident that super-resolution was not achieved with the proposed method (i.e., certainly the number of parameters parameterizing phase interface is larger than the number of pixels in the low-resolution image). Indeed, there are fine features, present in the object images but not reconstructed. Badly reconstructed area, although relatively small, is nonzero. The increase of quality, comparing to thresholding methods, was however achieved with the number of unknowns 100 times larger $(\mathrm{N}=10)$ than the number of equations.

\section{REFERENCES}

Elmoutaouakkil A, Peyrin F, Elkafi J, Laval-Jeantet AM (2002). Segmentation of cancellous bone from highresolution computed tomography images: influence on trabecular bone measurements. IEEE Trans Med Imaging 21:354-62.
Huiskes R, Ruimerman R, van Lenthe GH, Janssen GH (2000). Effects of mechanical forces on maintenance of form in trabecular bone. Nature 405:704-6.

Müller R, Koller B, Hildebrand T, Laib A, Gianolini S, Rüesegger P (1996). Resolution dependency of microstructural properties of cancellous bone based of threedimensional $\mu$-tomography. Technol Health Care 4: 113-9.

Park CP, Park MK, Kang MG (2003). Super-resolution image reconstruction: a technical overview. IEEE Signal Processing Magazine, May 2003:21-36.

Ross JC (1994). The Image Proccesing Handbook. Boca Raton: CRC Press.

Tabor Z, Rokita E (2000). Comparison of trabecular bone architecture in young and old bones. Med Phys 27: 1165-73.

Tabor Z, Rokita E, Cichocki T (2002). Origin of the pattern of trabecular bone: an experiment and a model. Phys Rev E 66:051906.

Ulrich D, van Rietbergen B, Weinans H, Rüegsegger P (1998). Finite element analysis of trabecular bone structure: a comparison of image based meshing techniques. J Biomech 31:1187-92.

van Rietbergen B, Weinans $\mathrm{H}$, Huiskes R, Odgaard A (1995). A new method to determine trabecular bone elastic properties and loading using micromechanical finite-elements models. J Biomech 28:69-81. 